\title{
ON THE PROBLEM OF UNCOUPLING SYSTEMS OF LINEAR DIFFERENTIAL EQUATIONS
}

\author{
JAMES M. HILL ${ }^{1}$ AND ALEX MCNABB ${ }^{2}$
}

(Received 22 December 1987; revised 23 June 1988)

\begin{abstract}
In modelling phenomena involving diffusion and chemical reactions, coupled systems of linear differential equations are often obtained, which can involve several dependent variables. For two dependent variables, coupled reaction-diffusion systems can be uncoupled, and in principle the original boundary value problem can be reduced to two separate boundary value problems for the classical heat equation. Here we address various aspects of the fundamental unsolved problem of the determination of corresponding uncoupling transformations for systems involving several dependent variables. We present, in an elementary manner, the current state of knowledge relating to this complex problem area. Several new results are obtained here. For example, in reviewing known results for two dependent variables we observe that those systems for which uncoupling transformations have been found are essentially those which can be reduced to a coupled system involving a single spatial operator $L$. In addition, for several dependent variables, the general solution structure for the kernel matrix, involved in the uncoupling transformation, is presented together with some explicit results for values of components of the kernel matrix along characteristics, which are deduced from elementary considerations.
\end{abstract}

\section{Introduction}

Multi-component systems undergoing diffusion and chemical reactions frequently give rise to coupled systems of linear differential equations involving several dependent variables. For example, for three diffusing species undergoing first-order chemical reactions we have the following coupled system for the concentrations

\footnotetext{
${ }^{1}$ Department of Mathematics, University of Wollongong, Wollongong, N.S.W. 2500, Australia. ${ }^{2}$ Department of Mathematics and Statistics, Massey University, Palmerston North, N.Z.

(C) Copyright Australian Mathematical Society 1989, Serial-fee code 0334-2700/89
} 
$c_{j}(\mathbf{x}, t)(j=1,2,3)$,

$$
\begin{aligned}
& \partial c_{1} / \partial t=d_{1} \nabla^{2} c_{1}+a_{11} c_{1}+a_{12} c_{2}+a_{13} c_{3} \\
& \partial c_{2} / \partial t=d_{2} \nabla^{2} c_{1}+a_{21} c_{1}+a_{22} c_{2}+a_{23} c_{3} \\
& \partial c_{3} / \partial t=d_{3} \nabla^{2} c_{3}+a_{31} c_{1}+a_{32} c_{2}+a_{33} c_{3}
\end{aligned}
$$

where $\nabla^{2}$ denotes the usual spatial Laplacian, the diffusivities $d_{j}(j=1,2,3)$ are positive constants which we suppose are such that $d_{1}>d_{2}>d_{3}$, and the matrix $\boldsymbol{A}=\left[a_{i j}\right]$ is assumed here to be a constant matrix with all components nonzero. In this paper we address the unsolved problem of finding transformations which uncouple systems such as (1.1) and in particular we seek uncoupling transformations which are independent of the spatial operator $\nabla^{2}$ involved in (1.1). Thus for any linear spatial operator $L$, involving partial derivatives of any order, we wish to uncouple the system,

$$
\begin{aligned}
& \partial c_{1} / \partial t=d_{1} L\left(c_{1}\right)+a_{11} c_{1}+a_{12} c_{2}+a_{13} c_{3} \\
& \partial c_{2} / \partial t=d_{2} L\left(c_{2}\right)+a_{21} c_{1}+a_{22} c_{2}+a_{23} c_{3} \\
& \partial c_{3} / \partial t=d_{3} L\left(c_{3}\right)+a_{31} c_{1}+a_{32} c_{2}+a_{33} c_{3} .
\end{aligned}
$$

For two dependent variables, this problem has been solved. Hill [2] shows that the coupled equations

$$
\begin{aligned}
& \partial c_{1} / \partial t=d_{1} L\left(c_{1}\right)+a_{11} c_{1}+a_{12} c_{2}, \\
& \partial c_{2} / \partial t=d_{2} L\left(c_{2}\right)+a_{21} c_{1}+a_{22} c_{2},
\end{aligned}
$$

where $L$ is any linear spatial operator, admit formal solutions of the form

$$
\begin{aligned}
c_{1}(\mathbf{x}, t)= & e^{a_{11} t} h_{1}\left(\mathbf{x}, d_{1} t\right) \\
& +\frac{e^{\lambda t}}{\left(d_{1}-d_{2}\right)} \int_{d_{2} t}^{d_{1} t} e^{-\mu \xi}\left\{\left(\frac{a_{12} a_{21}\left(\xi-d_{2} t\right)}{\left(d_{1} t-\xi\right)}\right)^{1 / 2} I_{1}(\eta) h_{1}(\mathbf{x}, \xi)\right. \\
& \left.+a_{12} I_{0}(\eta) h_{2}(\mathbf{x}, \xi)\right\} d \xi, \\
c_{2}(\mathbf{x}, t)= & e^{a_{22} t} h_{2}\left(\mathbf{x}, d_{2} t\right) \\
& +\frac{e^{\lambda t}}{\left(d_{1}-d_{2}\right)} \int_{d_{2} t}^{d_{1} t} e^{-\mu \xi}\left\{\left(\frac{a_{12} a_{21}\left(d_{1} t-\xi\right)}{\left(\xi-d_{2} t\right)}\right)^{1 / 2} I_{1}(\eta) h_{2}(\mathbf{x}, \xi)\right. \\
& \left.+a_{21} I_{0}(\eta) h_{1}(\mathbf{x}, \xi)\right\} d \xi,
\end{aligned}
$$

where the constants $\lambda$ and $\mu$ are given by

$$
\lambda=\left(\frac{d_{1} a_{22}-d_{2} a_{11}}{d_{1}-d_{2}}\right), \quad \mu=\left(\frac{a_{22}-a_{11}}{d_{1}-d_{2}}\right),
$$


and $I_{0}$ and $I_{1}$ are modified Bessel functions with $\eta$ defined by

$$
\eta=2 \frac{\left(a_{12} a_{21}\right)^{1 / 2}}{\left(d_{1}-d_{2}\right)}\left[\left(d_{1} t-\xi\right)\left(\xi-d_{2} t\right)\right]^{1 / 2} .
$$

Further $h_{1}(\mathrm{x}, t)$ and $h_{2}(\mathrm{x}, t)$ are understood to be solutions of

$$
\partial h / \partial t=L(h),
$$

satisfying the same initial conditions as $c_{1}(\mathbf{x}, t)$ and $c_{2}(\mathbf{x}, t)$ respectively. This result is important because, for example for coupled reaction-diffusion equations, certain boundary value problems can be reduced to similar boundary value problems but for the classical diffusion equation (see Hill [2]). The extension of this result to more than two dependent variables would have many important applications in applied mathematics. The original derivation of the formal solutions (1.4) (Aifantis and Hill [1] and Hill and Aifantis [3]) depends on formal mathematical devices such as Laplace and Fourier transforms and the success of this method hinges on the simple form of solutions of quadratic equations. For three dependent variables the method involves the solution of a cubic equation and the subsequent analysis proves intractable (see Lee [4]).

However $\mathrm{McNabb}$ [7] identified the essential structure of the uncoupling transformation (1.4) for any number of dependent variables and showed that the uncoupling problem hinges on solving a first order coupled system for a kernel matrix $k(t, s)$. Unfortunately the problem of the determination of this kernel matrix is also difficult and the purpose of this paper is to provide an elementary presentation of this problem and to address various aspects which are not explicitly discussed in $\mathrm{McNabb}$ [7]. In addition, we present one or two explicit results which are deduced from elementary considerations.

In the following section we first present a review of known results for coupled systems involving only two dependent variables. We show that those systems for which an uncoupling transformation has been found are essentially those which can be transformed into a coupled system (1.3) involving a single linear spatial operator $L$ only. In Section 3 we give the basic equations underlying the uncoupling procedure of $\mathrm{McNabb}$ [7] and we detail the general solution structure for the two kernel matrices $k^{+}(t, s)$ and $k^{-}(t, s)$. In Section 4 we then use invariance under a one-parameter group of transformations to give a brief derivation of the known kernels for the case of two dependent variables, and from these explicit expressions we deduce special values of the kernels along characteristics and show that the same special values can also be derived directly. Motivated by these results for the case of two dependent variables, we obtain corresponding expressions for three and more dependent variables in Sections 5 and 6 of the paper respectively. We remark that while systems such as (1.1) can be formulated as abstract evolution equations, for which the Hille-Yosida theorem provides sufficient conditions for a semi-group solution, unfortunately the verification of such 
conditions can be at least as complicated as the process of obtaining explicit results. For the semigroup approach we refer the reader to Pazy [9].

\section{Review of known results for two dependent variables}

In this section we show how other uncoupling transformations for two dependent variables (see Lee and Hill $[5,6]$ ) can also be derived from the basic results $(1.3)-(1.7)$ by transformations of the given system. In the first example (Lee and Hill [5]) we consider two coupled reaction-diffusion equations with convective terms, namely

$$
\begin{aligned}
& \partial c_{1} / \partial t+v_{1} \partial c_{1} / \partial x=d_{1} \partial^{2} c_{1} / \partial x^{2}+a_{11} c_{1}+a_{12} c_{2} \\
& \partial c_{2} / \partial t+v_{2} \partial c_{2} / \partial x=d_{2} \partial^{2} c_{2} / \partial x^{2}+a_{21} c_{1}+a_{22} c_{2}
\end{aligned}
$$

where the constants $d_{j}, a_{i j}(i, j=1,2)$ are as before and $v_{1}$ and $v_{2}$ are further constants. On introducing constants $\rho$ and $\sigma$ defined by

$$
\rho=\left(v_{1}-v_{2}\right) /\left(d_{1}-d_{2}\right), \quad \sigma=\left(v_{1} d_{2}-v_{2} d_{1}\right) /\left(d_{1}-d_{2}\right),
$$

and noting the relations,

$$
v_{1}=d_{1} \rho-\sigma, \quad v_{2}=d_{2} \rho-\sigma,
$$

we see that the system (2.1) becomes

$$
\frac{\partial c_{1}}{\partial t}-\sigma \frac{\partial c_{1}}{\partial x}=d_{1} L\left(c_{1}\right)+a_{11} c_{1}+a_{12} c_{2}, \quad \frac{\partial c_{2}}{\partial t}-\sigma \frac{\partial c_{2}}{\partial x}=d_{2} L\left(c_{2}\right)+a_{21} c_{1}+a_{22} c_{2},
$$

where here the operator $L$ is

$$
L=\frac{\partial^{2}}{\partial x^{2}}-\rho \frac{\partial}{\partial x} .
$$

Accordingly if we make the transformation

$$
x^{\prime}=x+\sigma t, \quad t^{\prime}=t
$$

then using

$$
\partial / \partial t=\partial / \partial t^{\prime}+\sigma \partial / \partial x^{\prime}, \quad \partial / \partial x=\partial / \partial x^{\prime},
$$

and from (2.4) we see that the coupled system finally becomes

$$
\frac{\partial c_{1}}{\partial t^{\prime}}=d_{1} L^{\prime}\left(c_{1}\right)+a_{11} c_{1}+a_{12} c_{2}, \quad \frac{\partial c_{2}}{\partial t^{\prime}}=d_{2} L^{\prime}\left(c_{2}\right)+a_{21} c_{1}+a_{22} c_{2},
$$

where the operator $L^{\prime}$ is given by

$$
L^{\prime}=\frac{\partial^{2}}{\partial x^{\prime 2}}-\rho \frac{\partial}{\partial x^{\prime}}
$$

Thus in this instance, we have by transformation of the independent variables, transformed (2.1) into a coupled system of the form (1.3) and the formal solutions 
(1.4)-(1.7) coincide with those derived in Lee and Hill [5], which were originally derived using formal techniques of mathematical analysis. In the second example we transform the coupled system into (1.3) by a transformation of the dependent variables.

Following the notation of Lee and Hill [6] we consider the reaction-diffusion system with cross-effects, that is

$$
\begin{aligned}
& \partial u_{1} / \partial t=D_{1} \nabla^{2} u_{1}+E_{1} \nabla^{2} u_{2}-A_{1} u_{1}+B_{1} u_{2} \\
& \partial u_{2} / \partial t=D_{2} \nabla^{2} u_{2}+E_{2} \nabla^{2} u_{1}-A_{2} u_{2}+B_{2} u_{1}
\end{aligned}
$$

where $A_{j}, B_{j}, D_{j}$ and $E_{j}(j=1,2)$ are all constants. Thus with

$$
\mathbf{u}=\left(\begin{array}{l}
u_{1} \\
u_{2}
\end{array}\right)
$$

the system (2.10) becomes

$$
\frac{\partial \mathbf{u}}{\partial t}=\left(\begin{array}{cc}
D_{1} & E_{1} \\
E_{2} & D_{2}
\end{array}\right) \nabla^{2} \mathbf{u}+\left(\begin{array}{cc}
-A_{1} & B_{1} \\
B_{2} & -A_{2}
\end{array}\right) \mathbf{u} .
$$

We now look for a transformation of dependent variables

$$
\mathbf{u}=T \mathbf{c}=\left(\begin{array}{ll}
a & b \\
c & d
\end{array}\right)\left(\begin{array}{l}
c_{1} \\
c_{2}
\end{array}\right)
$$

such that

$$
T^{-1}\left(\begin{array}{cc}
D_{1} & E_{1} \\
E_{2} & D_{2}
\end{array}\right) T=\left(\begin{array}{cc}
d_{1} & 0 \\
0 & d_{2}
\end{array}\right)
$$

where the elements of the matrix $T$ and $d_{j}(j=1,2)$ are all constants. If $(2.14)$ holds then (2.12) becomes

$$
\frac{\partial \mathrm{c}}{\partial t}=\left(\begin{array}{cc}
d_{1} & 0 \\
0 & d_{2}
\end{array}\right) \nabla^{2} \mathrm{c}+T^{-1}\left(\begin{array}{cc}
-A_{1} & B_{1} \\
B_{2} & -A_{2}
\end{array}\right) T \mathrm{c}
$$

which is a coupled system of the form (1.3). Undertaking the necessary algebra it is a simple matter to show that

$$
\begin{aligned}
d_{1} & =\left\{\left(D_{1}+D_{2}\right)+\left[\left(D_{1}-D_{2}\right)^{2}+4 E_{1} E_{2}\right]^{1 / 2}\right\} / 2 \\
d_{2} & =\left\{\left(D_{1}+D_{2}\right)-\left[\left(D_{1}-D_{2}\right)^{2}+4 E_{1} E_{2}\right]^{1 / 2}\right\} / 2 \\
a_{11} & =-\frac{1}{2}\left\{\left(A_{1}+A_{2}\right)+\frac{\left[\left(A_{1}-A_{2}\right)\left(D_{1}-D_{2}\right)-2\left(B_{1} E_{2}+B_{2} E_{1}\right)\right]}{\left[\left(D_{1}-D_{2}\right)^{2}+4 E_{1} E_{2}\right]^{1 / 2}}\right\} \\
a_{12} & =-\frac{d}{c}\left(\frac{D_{1}-d_{1}}{d_{1}-d_{2}}\right)\left\{\left(A_{1}-A_{2}\right)+\frac{B_{1} E_{2}}{\left(D_{2}-d_{2}\right)}-\frac{B_{2} E_{1}}{\left(D_{1}-d_{2}\right)}\right\} \\
a_{21} & =-\frac{c}{d}\left(\frac{D_{2}-d_{1}}{d_{1}-d_{2}}\right)\left\{\left(A_{1}-A_{2}\right)+\frac{B_{1} E_{2}}{\left(D_{2}-d_{1}\right)}-\frac{B_{2} E_{1}}{\left(D_{1}-d_{1}\right)}\right\} \\
a_{22} & =-\frac{1}{2}\left\{\left(A_{1}+A_{2}\right)-\frac{\left[\left(A_{1}-A_{2}\right)\left(D_{1}-D_{2}\right)-2\left(B_{1} E_{2}+B_{2} E_{1}\right)\right]}{\left[\left(D_{1}-D_{2}\right)^{2}+4 E_{1} E_{2}\right]^{1 / 2}}\right\}
\end{aligned}
$$


where the constants $a$ and $b$ are related to the constants $c$ and $d$ respectively by the equations,

$$
\begin{aligned}
& a=-E_{1} c /\left(D_{1}-d_{1}\right)=-\left(D_{2}-d_{1}\right) c / E_{2}, \\
& b=-E_{1} d /\left(D_{1}-d_{2}\right)=-\left(D_{2}-d_{2}\right) d / E_{2} .
\end{aligned}
$$

We observe that $c$ and $d$ are undetermined arbitrary constants and that in the notation of Lee and Hill [6] we have $a_{11}=-\nu_{1}$ and $a_{22}=-\nu_{2}$. Further, on making repeated use of the relation $d_{1}+d_{2}=D_{1}+D_{2}$, we can show from (2.16) that the quantity $k=a_{12} a_{21}$ arising in (1.6) becomes

$$
\begin{aligned}
k= & {\left[E_{1} E_{2}\left(A_{1}-A_{2}\right)^{2}+B_{1} B_{2}\left(D_{1}-D_{2}\right)^{2}-\left(B_{1} E_{2}-B_{2} E_{1}\right)^{2}\right.} \\
& \left.+\left(A_{1}-A_{2}\right)\left(D_{1}-D_{2}\right)\left(B_{1} E_{2}+B_{2} E_{1}\right)\right] /\left[\left(D_{1}-D_{2}\right)^{2}+4 E_{1} E_{2}\right],
\end{aligned}
$$

which is precisely as given in Lee and Hill [6]. Thus, assuming we can transform (2.10) into a coupled system of the form (1.3), the formal solutions (1.4) are "essentially" those given in Lee and Hill [6], which were obtained by an alternative procedure. However the two sets of solutions are not identical, since those arising from (1.4) have an initial condition coinciding with that of $c_{j}(\mathbf{x}, t)(j=1,2)$ while those derived in Lee and Hill [6] have an initial condition coinciding with that of $u_{j}(\mathbf{x}, t)(j=1,2)$.

An important unsolved problem would be to obtain formal solutions (1.4)(1.7) for coupled systems of the form (1.3) but with distinct linear spatial operators $L_{1}$ and $L_{2}$, namely

$$
\partial c_{1} / \partial t=d_{1} L_{1}\left(c_{1}\right)+a_{11} c_{1}+a_{12} c_{2}, \quad \partial c_{2} / \partial t=d_{2} L_{2}\left(c_{2}\right)+a_{21} c_{1}+a_{22} c_{2}
$$

A more restricted version of this problem would be to obtain formal solutions for a coupled system involving linear spatial operators $L$ and $M$ and being of the form,

$$
\begin{aligned}
& \partial c_{1} / \partial t=d_{1} L\left(c_{1}\right)+e_{1} M\left(c_{1}\right)+a_{11} c_{1}+a_{12} c_{2}, \\
& \partial c_{2} / \partial t=d_{2} L\left(c_{2}\right)+e_{2} M\left(c_{2}\right)+a_{21} c_{1}+a_{22} c_{2},
\end{aligned}
$$

where $e_{j}(j=1,2)$ are constants. In the remaining sections of the paper we focus on the problem of obtaining uncoupling transformations for systems involving several dependent variables.

\section{Basic equations for the kernel matrix}

McNabb [7] showed that solutions of the coupled system

$$
\partial \mathbf{c} / \partial t=D L(\mathbf{c})+A \mathbf{c},
$$


where $D=\left[d_{j}\right]$ is a positive constant diagonal matrix and $A=\left[a_{i j}\right]$ is a general matrix not involving spatial variables, can be expressed in terms of solutions of the uncoupled system

$$
\partial \mathbf{H} / \partial t=D L(\mathbf{H})
$$

by means of relations of the form,

$$
\begin{aligned}
\mathbf{c}(\mathbf{x}, t) & =(J+K) \mathbf{H}(\mathbf{x}, t) \\
& =J \mathbf{H}(\mathbf{x}, t)+\int_{0}^{t} k^{+}(t, s) \mathbf{H}(\mathbf{x}, s) d s+\int_{t}^{\infty} k^{-}(t, s) \mathbf{H}(\mathbf{x}, s) d s
\end{aligned}
$$

Here for simplicity we assume at the outset firstly that the elements of $D$ are distinct and the variables are labelled such that

$$
d_{1}>d_{2}>d_{3} \ldots d_{n}
$$

and secondly that the matrix $A$ is a constant matrix with all elements nonzero. In these circumstances the matrix $J$ in (3.3) is diagonal and given simply by

$$
J(t)=\left[\exp \left(a_{j j} t\right)\right]
$$

Further the kernel matrices $k^{+}(t, s)$ and $k^{-}(t, s)$ are obtained as solutions of the hyperbolic equations

$$
\begin{array}{ll}
\frac{\partial k^{+}}{\partial t} D+D \frac{\partial k^{+}}{\partial s}=A k^{+} D, & 0 \leq t \leq \infty, 0 \leq s \leq t \\
\frac{\partial k^{-}}{\partial t} D+D \frac{\partial k^{-}}{\partial s}=A k^{-} D, & 0 \leq s \leq \infty, 0 \leq t \leq s
\end{array}
$$

with boundary conditions

$$
k^{+}(t, 0)=k^{-}(0, s)=0,
$$

and jump conditions along $s=t$,

$$
\left[k^{+}(t, t)-k^{-}(t, t)\right] D-D\left[k^{+}(t, t)-k^{-}(t, t)\right]=\left(A-A^{0}\right) J D,
$$

where with the above assumptions $A^{0}$ is simply the diagonal matrix $\left[a_{i j}\right]$.

In component form, with $k^{+}(t, s)=\left[k_{i j}^{+}(t, s)\right]$ and $k^{-}(t, s)=\left[k_{i j}^{-}(t, s)\right]$ the above equations become for $1 \leq i, j \leq n$,

$$
\begin{gathered}
d_{j} \frac{\partial k_{i j}^{+}}{\partial t}+d_{i} \frac{\partial k_{i j}^{+}}{\partial s}=\sum_{l=1}^{n} a_{\imath l} d_{j} k_{l j}^{+}, \quad d_{j} \frac{\partial k_{i j}^{-}}{\partial t}+d_{i} \frac{\partial k_{i j}^{-}}{\partial s}=\sum_{l=1}^{n} a_{i l} d_{j} k_{l j}^{-}, \\
k_{i j}^{+}(t, 0)=k_{i j}^{-}(0, s)=0, \\
{\left[k_{i j}^{+}(t, t)-k_{i j}^{-}(t, t)\right]\left(d_{j}-d_{i}\right)=a_{i j} d_{j} e^{a_{j j} t} \quad(i \neq j) .}
\end{gathered}
$$

We make the following observations relating to the solution of (3.9)-(3.11). Clearly $k_{i j}^{+}$and $k_{i j}^{-}$satisfy the same system of first-order partial differential 
equations and in principle both are solved column by column; that is, the coupling in (3.9) arises only through elements of the kernel matrix which are in the same column. Further, the character of the solutions $k_{i j}^{+}$and $k_{i j}^{-}$is dictated by the slope of the characteristics of (3.9), which are given by

$$
s-d_{i} t / d_{j}=\text { constant } .
$$

Thus if we consider the problem of solving for the $j$ th column, then because of (3.4) we have $d_{i} / d_{j}>1$ for $1 \leq i \leq j-1$ while $d_{i} / d_{j}<1$ for $j+1 \leq i \leq n$. Further, the largest ratio is $d_{1} / d_{j}$ and the smallest is $d_{n} / d_{j}$, which means that the kernel matrices are nonzero only in the fan contained by $s=d_{n} t / d_{j}$ and $s=d_{1} t / d_{j}$. In particular $k_{i j}^{+}$is nonzero only in the fan contained by $s=d_{n} t / d_{j}$ and $s=t$, while $k_{i j}^{-}$is nonzero only in the fan contained by $s=t$ and $s=d_{1} t / d_{j}$.

For the first column of the kernel matrices $(j=1)$ we have $d_{i} / d_{1} \leq 1$ for all $i$ and therefore $k_{i 1}^{-}(t, s)$ is identically zero for $1 \leq i \leq n$. On the other hand, for the last column of the kernel matrices $(j=n)$ we have $d_{\imath} / d_{n} \geq 1$ for all $i$, and therefore $k_{\text {in }}^{+}(t, s)$ is identically zero for $1 \leq i \leq n$. For these two special columns, the jump conditions along $s=t$ become

$$
\begin{array}{ll}
k_{i 1}^{+}(t, t)=a_{i 1} e^{a_{11} t} /\left(1-d_{i} / d_{1}\right) & (2 \leq i \leq n), \\
k_{i n}^{-}(t, t)=a_{i n} e^{a_{n n} t} /\left(d_{i} / d_{n}-1\right) & (1 \leq i \leq n-1) .
\end{array}
$$

Incorporating these details in (3.3) gives

$$
\begin{aligned}
c_{i}(\mathbf{x}, t)= & e^{a_{i i} t} H_{i}(\mathbf{x}, t) \\
& +\sum_{j=1}^{n-1} \int_{d_{n} t / d_{j}}^{t} k_{i j}^{+}(t, s) H_{j}(\mathbf{x}, s) d s+\sum_{j=2}^{n} \int_{t}^{d_{1} t / d,} k_{\imath j}^{-}(t, s) H_{j}(\mathbf{x}, s) d s .
\end{aligned}
$$

Thus the underlying feature when solving (3.9)-(3.11) for either of the kernel matrices $k^{+}(t, s)$ or $k^{-}(t, s)$ is that either all elements of the column are identically zero or they are all nonzero. Clearly for certain special matrices $A$ with some zero elements this may not be the case. Our discussion pertains to the general matrix $A$ with all elements nonzero, so that once one element of the column of the kernel matrices is nonzero the coupling in (3.9) generates nonzero elements throughout the column. Finally in this section we make the observation that when solving for the $j$ th column of the kernel matrices the elements of $k_{i j}^{+}(t, s)$ and $k_{i j}^{-}(t, s)$ are continuous functions across all characteristics except their own characteristic, namely $s=d_{i} t / d_{j}$. If we introduce $\Delta_{i j} k_{\alpha \beta}^{ \pm}(t)$ as the discontinuity of $k_{\alpha \beta}^{ \pm}$across the characteristic $s=d_{i} t / d_{j}$, that is

$$
\Delta_{\imath \jmath} k_{\alpha \beta}^{ \pm}(t)=k_{\alpha \beta}^{ \pm}\left(t^{+}, d_{\imath} t / d_{\jmath}\right)-k_{\alpha \beta}^{ \pm}\left(t^{-}, d_{i} t / d_{\jmath}\right),
$$

then we are claiming that

$$
\Delta_{i j} k_{\alpha \beta}^{ \pm}(t)=0
$$


unless $d_{\alpha} / d_{\beta}=d_{i} / d_{j}$. This means, for example, that when solving for the $j$ th column of $k^{+}(t, s)$, all elements are zero in the fan contained by $s=0$ and $s=$ $d_{n} t / d_{j}$ and then $k_{n j}^{+}(t, s)$ suffers a jump across $s=d_{n} t / d_{j}$ and becomes nonzero. Because of the coupling this nonzero element generates further nonzero elements of the $j$ th column. These preliminary remarks enable us to make appropriate interpretation of the known kernel matrices for two variables which lead to one or two explicit results for several variables which we show can also be deduced from (3.9)-(3.11) by elementary arguments.

\section{Derivation of known kernel matrix for two dependent variables}

In this section we present a slightly different derivation of the kernel matrices for two variables to that given by $\mathrm{McNabb}$ [7]. From (3.9) we find that the first column of the kernel matrices $k^{+}(t, s)$ and $k^{-}(t, s)$ both satisfy

$$
\frac{\partial k_{11}}{\partial t}+\frac{\partial k_{11}}{\partial s}=a_{11} k_{11}+a_{12} k_{21}, \quad \frac{\partial k_{21}}{\partial t}+\frac{d_{2}}{d_{1}} \frac{\partial k_{21}}{\partial s}=a_{21} k_{11}+a_{22} k_{21} .
$$

Now since $d_{2} / d_{1}<1$, the elements $k_{11}^{-}(t, s)$ and $k_{21}^{-}(t, s)$ are identically zero in $0 \leq t \leq s$. Hence we confine our attention to $k^{+}(t, s)$ and from (3.10) and (3.13) we require that

$$
k_{11}^{+}(t, 0)=k_{21}^{+}(t, 0)=0, \quad k_{21}^{+}(t, t)=a_{21} e^{a_{11} t} /\left(1-d_{2} / d_{1}\right) .
$$

Now $k_{11}^{+}(t, s)$ and $k_{21}^{+}(t, s)$ are zero in the fan contained by $s=0$ and $s=d_{2} t / d_{1}$ and on the line $s=d_{2} t / d_{1}, k_{21}^{+}(t, s)$ suffers a jump while $k_{11}^{-}(t, s)$ is continuous across this line. It can be seen after a little thought that the natural variables of the problem are,

$$
x=\left(s-d_{2} t / d_{1}\right) /\left(1-d_{2} / d_{1}\right), \quad y=(t-s) /\left(1-d_{2} / d_{1}\right),
$$

because in these coordinates (4.1) becomes

$$
\partial k_{11}^{+} / \partial x=a_{11} k_{11}^{+}+a_{12} k_{21}^{+}, \quad \partial k_{21}^{+} / \partial y=a_{21} k_{11}^{+}+a_{22} k_{21}^{+},
$$

and on the line $s=d_{2} t / d_{1}$ we have

$$
x=0, \quad y=t, \quad k_{11}^{+}=0,
$$

while on the line $s=t$ we have

$$
x=t, \quad y=0, \quad k_{21}^{+}=a_{21} e^{a_{11} t} /\left(1-d_{2} / d_{1}\right) .
$$

We now make the transformation

$$
k_{11}^{+}=e^{a_{11} x+a_{22} y} \phi_{11}(x, y), \quad k_{21}^{+}=e^{a_{11} x+a_{22} y} \phi_{21}(x, y),
$$


so that (4.4)-(4.6) give

$$
\begin{gathered}
\partial \phi_{11} / \partial x=a_{12} \phi_{21}, \quad \partial \phi_{21} / \partial y=a_{21} \phi_{11} \\
\phi_{11}(0, y)=0, \quad \phi_{21}(x, 0)=a_{21} /\left(1-d_{2} / d_{1}\right)
\end{gathered}
$$

and it is not difficult to see that this problem remains invariant under the oneparameter group of transformations

$$
x^{*}=e^{\varepsilon} x, \quad y^{*}=e^{-\varepsilon} y, \quad \phi_{11}^{*}=e^{\varepsilon} \phi_{11}, \quad \phi_{21}^{*}=\phi_{21} .
$$

Accordingly our solution takes the form

$$
\phi_{11}(x, y)=x \Phi_{11}(x y), \quad \phi_{21}(x, y)=\Phi_{21}(x y) .
$$

It is now a straightforward matter to deduce

$$
\begin{gathered}
k_{11}^{+}(t, s)=\frac{e^{a_{11} x+a_{22} y}}{1-d_{2} / d_{1}}\left(a_{12} a_{21} x / y\right)^{1 / 2} I_{1}\left[2\left(a_{12} a_{21} x y\right)^{1 / 2}\right] \\
k_{21}^{+}(t, s)=\frac{e^{a_{11} x+a_{22} y}}{1-d_{2} / d_{1}} a_{21} I_{0}\left[2\left(a_{12} a_{21} x y\right)^{1 / 2}\right]
\end{gathered}
$$

which coincide with the known expressions given in (1.4).

Similarly for the second column of the kernel matrices $k^{+}(t, s)$ and $k^{-}(t, s)$ we need to solve

$$
\frac{\partial k_{12}}{\partial t}+\frac{d_{1}}{d_{2}} \frac{\partial k_{12}}{\partial s}=a_{11} k_{12}+a_{12} k_{22}, \quad \frac{\partial k_{22}}{\partial t}+\frac{\partial k_{22}}{\partial s}=a_{21} k_{12}+a_{22} k_{22},
$$

and in this case, because $d_{1} / d_{2}>1, k_{12}^{+}(t, s)$ and $k_{22}^{+}(t, s)$ are identically zero in the region $0 \leq s \leq t$ and proceeding exactly as above but with coordinates

$$
X=(s-t) /\left(d_{1} / d_{2}-1\right), \quad Y=\left(d_{1} t / d_{2}-s\right) /\left(d_{1} / d_{2}-1\right),
$$

we require to solve

$$
\partial k_{12}^{-} / \partial X=a_{11} k_{12}^{-}+a_{12} k_{22}^{-}, \quad \partial k_{22}^{-} / \partial Y=a_{21} k_{12}^{-}+a_{22} k_{22}^{-},
$$

such that on the line $s=d_{1} t / d_{2}$ we have

$$
X=t, \quad Y=0, \quad k_{22}^{-}=0,
$$

while on the line $s=t$ we have

$$
X=0, \quad Y=t, \quad k_{12}^{-}=a_{12} e^{a_{22} t} /\left(d_{1} / d_{2}-1\right) .
$$

We may readily deduce that

$$
\begin{gathered}
k_{12}^{-}(t, s)=\frac{e^{a_{11} X+a_{22} Y}}{\left(d_{1} / d_{2}-1\right)} a_{12} I_{0}\left[2\left(a_{12} a_{21} X Y\right)^{1 / 2}\right] \\
k_{22}^{-}(t, s)=\frac{e^{a_{11} X+a_{22} Y}}{\left(d_{1} / d_{2}-1\right)}\left(a_{12} a_{21} Y / X\right)^{1 / 2} I_{1}\left[2\left(a_{12} a_{21} X Y\right)^{1 / 2}\right] .
\end{gathered}
$$


The solutions (4.11) and (4.17) satisfy the appropriate boundary data but in addition we may readily confirm the following,

$$
\begin{array}{ll}
k_{11}^{+}(t, t)=e^{a_{11} t} a_{12} a_{21} t /\left(1-d_{2} / d_{1}\right), & k_{12}^{-}\left(t, d_{1} t / d_{2}\right)=e^{a_{11} t} a_{12} /\left(d_{1} / d_{2}-1\right) \\
k_{21}^{+}\left(t, d_{2} t / d_{1}\right)=e^{a_{22} t} a_{21} /\left(1-d_{2} / d_{1}\right), & k_{22}^{-}(t, t)=e^{a_{22} t} a_{12} a_{21} t /\left(d_{1} / d_{2}-1\right)
\end{array}
$$

It is of some interest that these results can also be derived from elementary considerations. For example for $k_{11}^{+}(t, t)$ we have from the first equation of (4.1)

$$
\frac{\partial k_{11}^{+}}{\partial t}(t, t)+\frac{\partial k_{11}^{+}}{\partial s}(t, t)=a_{11} k_{11}^{+}(t, t)+a_{12} k_{21}^{+}(t, t)
$$

which on using (4.2) becomes

$$
\left(\frac{d}{d t}-a_{11}\right) k_{11}^{+}(t, t)=a_{12} a_{21} e^{a_{11} t} /\left(1-d_{2} / d_{1}\right)
$$

where here $d / d t$ is the total derivative with respect to time along the line $s=t$. On integrating (4.20) we may readily deduce

$$
k_{11}^{+}(t, t)=e^{a_{11} t}\left\{a_{12} a_{21} t /\left(1-d_{2} / d_{1}\right)+C_{1}\right\}
$$

where $C_{1}$ denotes the constant of integration. Now as $t$ tends to zero we require that $k_{11}^{+}(0,0)$ is zero and therefore the constant $C_{1}$ is zero and we have deduced the given expression. Similarly for $k_{21}^{+}\left(t, d_{2} t / d_{1}\right)$ we have from the second equation of (4.1)

$$
\frac{\partial k_{21}^{+}}{\partial t}\left(t, d_{2} t / d_{1}\right)+\frac{d_{2}}{\partial_{1}} \frac{\partial k_{21}^{+}}{\partial s}\left(t, d_{2} t / d_{1}\right)=a_{22} k_{21}^{+}\left(t, d_{2} t / d_{1}\right)
$$

where we have used the fact that $k_{11}^{+}\left(t, d_{2} t / d_{1}\right)$ is zero. Now (4.22) becomes

$$
\left(\frac{d}{d t}-a_{22}\right) k_{21}^{+}\left(t, d_{2} t / d_{1}\right)=0
$$

which evidently has the solution

$$
k_{21}^{+}\left(t, d_{2} t / d_{1}\right)=C_{2} e^{a_{22} t}
$$

where the constant of integration $C_{2}$ is determined from the condition that the jump in $k_{21}^{+}$across $s=d_{2} t / d_{1}$ at the origin coincides with the known value deduced from the expression given in (4.2) as $t$ tends to zero, namely

$$
C_{2}=a_{21} /\left(1-d_{2} / d_{1}\right)
$$


which together with (4.24) yields the given expression. These simple results giving values of components of the kernel matrices along the extreme characteristics and along $s=t$ can be extended to the case of more than two dependent variables and this is done in the following sections.

\section{Kernel matrices for three dependent variables}

For $n=3$ we find from (3.9) that the first column of the kernel matrices $k^{+}(t, s)$ and $k^{-}(t, s)$ both satisfy

$$
\begin{gathered}
\frac{\partial k_{11}}{\partial t}+\frac{\partial k_{11}}{\partial s}=a_{11} k_{11}+a_{12} k_{21}+a_{13} k_{31} \\
\frac{\partial k_{21}}{\partial t}+\frac{d_{2}}{d_{1}} \frac{\partial k_{21}}{\partial s}=a_{21} k_{11}+a_{22} k_{21}+a_{23} k_{31} \\
\frac{\partial k_{31}}{\partial t}+\frac{d_{3}}{d_{1}} \frac{\partial k_{31}}{\partial s}=a_{31} k_{11}+a_{32} k_{21}+a_{33} k_{31}
\end{gathered}
$$

and since $d_{2} / d_{1}$ and $d_{3} / d_{1}$ are both less than unity the first column of $k^{-}(t, s)$ is identically zero. The first column of $k^{+}(t, s)$ is identically zero in the fan contained by $s=d_{3} t / d_{1}$ and $s=0$ and from (3.10) and (3.13) we have the special values,

$$
\begin{gathered}
k_{11}^{+}\left(t, d_{3} t / d_{1}\right)=k_{21}^{+}\left(t, d_{3} t / d_{1}\right)=0 \\
k_{21}^{+}(t, t)=a_{21} e^{a_{11} t} /\left(1-d_{2} / d_{1}\right), \quad k_{31}^{+}(t, t)=a_{31} e^{a_{11} t} /\left(1-d_{3} / d_{1}\right) .
\end{gathered}
$$

Now from the first equation of $(5.1)$ along the line $s=t$ and these special values we have

$$
\left(\frac{d}{d t}-a_{11}\right) k_{11}^{+}(t, t)=\left\{\frac{a_{12} a_{21}}{\left(1-d_{2} / d_{1}\right)}+\frac{a_{13} a_{31}}{\left(1-d_{3} / d_{1}\right)}\right\} e^{a_{11} t}
$$

which on integration and using $k_{11}^{+}(0,0)$ is zero gives as outlined in the previous section

$$
k_{11}^{+}(t, t)=\left\{\frac{a_{12} a_{21}}{\left(1-d_{2} / d_{1}\right)}+\frac{a_{13} a_{31}}{\left(1-d_{3} / d_{1}\right)}\right\} t e^{a_{11} t} .
$$

Further the last equation of (5.1) along the line $s=d_{3} t / d_{1}$ yields

$$
\left(\frac{d}{d t}-a_{33}\right) k_{31}^{+}\left(t, d_{3} t / d_{1}\right)=0
$$

so that

$$
k_{31}^{+}\left(t, d_{3} t / d_{1}\right)=a_{31} e^{a_{33} t} /\left(1-d_{3} / d_{1}\right),
$$

where the constant of integration has to be chosen so that $k_{31}^{+}(0,0)$ agrees with the value predicted by $(5.2)_{4}$. We observe that while the values of $k_{11}^{+}(t, t)$ and $k_{31}^{+}\left(t, d_{3} t / d_{1}\right)$ as given by (5.4) and (5.6) respectively have been deduced to be 
consistent with (5.2) as $t$ tends to zero, it is apparent from $(5.2)_{2}$ and $(5.2)_{3}$ that $k_{21}^{+}$is certainly not continuous at the origin.

Now since the first column of $k^{+}(t, s)$ is nonzero only in the fan contained by the lines $s=d_{3} t / d_{1}$ and $s=t$, the natural coordinates for this problem appear to be

$$
x=\left(s-d_{3} t / d_{1}\right) /\left(1-d_{3} / d_{1}\right), \quad y=(t-s) /\left(1-d_{3} / d_{1}\right),
$$

in which case $(5.1)$ becomes

$$
\begin{gathered}
\partial k_{11}^{+} / \partial x=a_{11} k_{11}^{+}+a_{12} k_{21}^{+}+a_{13} k_{31}^{+} \\
\alpha \partial k_{21}^{+} / \partial x+\beta \partial k_{21}^{+} / \partial y=a_{21} k_{11}^{+}+a_{22} k_{21}^{+}+a_{23} k_{31}^{+} \\
\partial k_{31}^{+} / \partial y=a_{31} k_{11}^{+}+a_{32} k_{21}^{+}+a_{33} k_{31}^{+}
\end{gathered}
$$

where the constants $\alpha$ and $\beta$ are defined by

$$
\alpha=\left(d_{2}-d_{3}\right) /\left(d_{1}-d_{3}\right), \quad \beta=\left(d_{1}-d_{2}\right) /\left(d_{1}-d_{3}\right),
$$

which we notice are such that $\alpha+\beta=1$. We now make the transformations

$$
\begin{aligned}
& k_{11}^{+}(t, s)=e^{a_{11} x+a_{33} y} \phi_{1}(x, y), \\
& k_{21}^{+}(t, s)=e^{a_{11} x+a_{33} y} \phi_{2}(x, y), \\
& k_{31}^{+}(t, s)=e^{a_{11} x+a_{33} y} \phi_{3}(x, y),
\end{aligned}
$$

so that $(5.8)$ becomes

$$
\begin{gathered}
\partial \phi_{1} / \partial x=a_{12} \phi_{2}+a_{13} \phi_{3} \\
\alpha \partial \phi_{2} / \partial x+\beta \partial \phi_{2} / \partial y-\Delta \phi_{2}=a_{21} \phi_{1}+a_{23} \phi_{3} \\
\partial \phi_{3} / \partial y=a_{31} \phi_{1}+a_{32} \phi_{2}
\end{gathered}
$$

where the constant $\Delta$ is defined by

$$
\Delta=a_{22}-\left(\alpha a_{11}+\beta a_{33}\right) .
$$

Further the boundary conditions (5.2), (5.4) and (5.6) become

$$
\begin{gathered}
\phi_{1}(x, 0)=\left\{a_{12} a_{21} /\left(1-d_{2} / d_{1}\right)+a_{13} a_{31} /\left(1-d_{3} / d_{1}\right)\right\} x \\
\phi_{2}(x, 0)=a_{21} /\left(1-d_{2} / d_{1}\right), \quad \phi_{3}(x, 0)=a_{31} /\left(1-d_{3} / d_{1}\right) \\
\phi_{1}(0, y)=\phi_{2}(0, y)=0, \quad \phi_{3}(0, y)=a_{31} /\left(1-d_{3} / d_{1}\right)
\end{gathered}
$$

Because of the zero conditions (5.13) 4 and $(5.13)_{5}$ and because partial differentiation with respect to $x$ only occurs in (5.11) for the functions $\phi_{1}$ and $\phi_{2}$, it is natural to Laplace transform (5.11) with respect to $x$. Although the resulting transforms are readily obtained, they are not of a type which can be 
readily inverted. Accordingly we do not pursue this line of investigation further. Alternatively from (5.11) and (5.13) we may deduce the following integral formulation,

$$
\begin{gathered}
\phi_{1}(x, y)=\int_{0}^{x}\left[a_{12} \phi_{2}(\tau, y)+a_{13} \phi_{3}(\tau, y)\right] d \tau \\
\phi_{2}(x, y)=\int_{0}^{x} \frac{e^{-\Delta(\tau-x) / \alpha}}{\alpha}\left[a_{21} \phi_{1}\left(\tau, y+\frac{\beta(\tau-x)}{\alpha}\right)\right. \\
\left.+a_{23} \phi_{3}\left(\tau, y+\frac{\beta(\tau-x)}{\alpha}\right)\right] d \tau \\
\phi_{3}(x, y)=\frac{a_{31}}{\left(1-d_{3} / d_{1}\right)}+\int_{0}^{x}\left[a_{31} \phi_{1}(x, \tau)+a_{32} \phi_{2}(x, \tau)\right] d \tau
\end{gathered}
$$

which possibly could form the basis of an iterative scheme for obtaining approximate kernels. This is actually the iterative scheme employed in $\mathrm{McNabb}$ [7] to prove existence.

From (3.9) the middle columns of $k^{+}(t, s)$ and $k^{-}(t, s)$ both satisfy

$$
\begin{gathered}
\frac{\partial k_{12}}{\partial t}+\frac{d_{1}}{d_{2}} \frac{\partial k_{12}}{\partial s}=a_{11} k_{12}+a_{12} k_{22}+a_{13} k_{32} \\
\frac{\partial k_{22}}{\partial t}+\frac{\partial k_{22}}{\partial s}=a_{21} k_{12}+a_{22} k_{22}+a_{23} k_{32} \\
\frac{\partial k_{32}}{\partial t}+\frac{d_{3}}{d_{2}} \frac{\partial k_{32}}{\partial s}=a_{31} k_{12}+a_{32} k_{22}+a_{33} k_{32}
\end{gathered}
$$

while from (3.10) and (3.11) we may deduce

$$
\begin{gathered}
k_{12}^{+}\left(t, d_{3} t / d_{2}\right)=k_{22}^{+}\left(t, d_{3} t / d_{2}\right)=0 \\
k_{12}^{+}(t, t)-k_{12}^{-}(t, t)=a_{12} e^{a_{22} t} /\left(1-d_{1} / d_{2}\right), \\
k_{32}^{+}(t, t)-k_{32}^{-}(t, t)=a_{32} e^{a_{22} t} /\left(1-d_{3} / d_{2}\right), \\
k_{22}^{-}\left(t, d_{1} t / d_{2}\right)=k_{32}^{-}\left(t, d_{1} t / d_{2}\right)=0 .
\end{gathered}
$$

From these special values and each of the equations (5.15), we may deduce exactly as previously described, the following additional special values,

$$
\begin{gathered}
k_{32}^{+}\left(t, d_{3} t / d_{2}\right)=-a_{32} e^{a_{33} t} /\left(1-d_{3} / d_{2}\right) \\
k_{22}^{+}(t, t)-k_{22}^{-}(t, t)=\left\{a_{21} a_{12} /\left(1-d_{1} / d_{2}\right)+a_{23} a_{32} /\left(1-d_{3} / d_{2}\right)\right\} t e^{a_{22} t} \\
k_{12}^{-}\left(t, d_{1} t / d_{2}\right)=a_{12} e^{a_{11} t} /\left(1-d_{1} / d_{2}\right) .
\end{gathered}
$$


Similarly the third columns of $k^{+}(t, s)$ and $k^{-}(t, s)$ both satisfy

$$
\begin{gathered}
\frac{\partial k_{13}}{\partial t}+\frac{d_{1}}{d_{3}} \frac{\partial k_{13}}{\partial s}=a_{11} k_{13}+a_{12} k_{23}+a_{13} k_{33} \\
\frac{\partial k_{23}}{\partial t}+\frac{d_{2}}{d_{3}} \frac{\partial k_{23}}{\partial s}=a_{21} k_{13}+a_{22} k_{23}+a_{23} k_{33} \\
\frac{\partial k_{33}}{\partial t}+\frac{\partial k_{33}}{\partial s}=a_{31} k_{13}+a_{32} k_{23}+a_{33} k_{33}
\end{gathered}
$$

and since the third column of $k^{+}(t, s)$ is identically zero we have from (3.10) and (3.13),

$$
\begin{gathered}
k_{23}^{-}\left(t, d_{1} t / d_{3}\right)=k_{33}^{-}\left(t, d_{1} t / d_{3}\right)=0, \\
k_{13}^{-}(t, t)=a_{13} e^{a_{33} t} /\left(d_{1} / d_{3}-1\right), \quad k_{23}^{-}(t, t)=a_{23} e^{a_{33} t} /\left(d_{2} / d_{3}-1\right),
\end{gathered}
$$

and in the usual way we may deduce the additional special values

$$
\begin{gathered}
k_{13}^{-}\left(t, d_{1} t / d_{3}\right)=-a_{13} e^{a_{11} t} /\left(d_{1} / d_{3}-1\right) \\
k_{33}^{-}(t, t)=\left\{\frac{a_{13} a_{31}}{\left(d_{1} / d_{3}-1\right)}+\frac{a_{23} a_{32}}{\left(d_{2} / d_{3}-1\right)}\right\} t e^{a_{33} t}
\end{gathered}
$$

Some of these results are extended to $n$ variables in the following section.

Finally in this section we note that McNabb [7] has shown that the Laplace transforms of the kernel matrices admit particularly simple forms in a matrix formulation. For example for the first column of $k^{+}(t, s)$ we have

$$
\hat{k}_{i 1}^{+}(t, p)=\int_{0}^{t} e^{-p s} k_{i 1}^{+}(t, s) d s
$$

and from (5.1) and (5.2) we may deduce

$$
\begin{aligned}
& d \hat{k}_{11}^{+} / d t=\left(a_{11}-p\right) \hat{k}_{11}^{+}+a_{12} \hat{k}_{21}^{+}+a_{13} \hat{k}_{31}^{+} \\
& d \hat{k}_{21}^{+} / d t=a_{21} \hat{k}_{11}^{+}+\left(a_{22}-p d_{2} / d_{1}\right) \hat{k}_{21}^{+}+a_{23} \hat{k}_{31}^{+}+a_{21} e^{\left(a_{11}-p\right) t} \\
& d \hat{k}_{31}^{+} / d t=a_{31} \hat{k}_{11}^{+}+a_{32} \hat{k}_{21}^{+}+\left(a_{33}-p d_{3} / d_{1}\right) \hat{k}_{31}^{+}+a_{31} e^{\left(a_{11}-p\right) t}
\end{aligned}
$$

From these equations we may readily deduce, in terms of matrices $A$ and $D$ the following seemingly simple expression,

$$
\left(\begin{array}{l}
\hat{k}_{11}^{+} \\
\hat{k}_{21}^{+} \\
\hat{k}_{31}^{+}
\end{array}\right)=\left\{e^{\left(A-p D / d_{1}\right) t}-e^{\left(a_{11}-p\right) t} I\right\}\left(\begin{array}{l}
1 \\
0 \\
0
\end{array}\right)
$$


where $I$ denotes the identity matrix. The highly nontrivial problem of inverting such transforms is considered in some detail by McNabb [8].

\section{Some elementary results for $n$ dependent variables}

For the first column of the kernel matrices $k^{+}$and $k^{-}$, all the elements of $k^{-}$ are zero while for $k^{+}$which is nonzero in the fan contained by $s=d_{n} t / d_{1}$ and $s=t$, we have from (3.9), (3.10) and $(3.13)_{1}$,

$$
\begin{gathered}
\frac{\partial k_{i 1}^{+}}{\partial t}+\frac{d_{i}}{d_{1}} \frac{\partial k_{i 1}^{+}}{\partial s}=\sum_{l=1}^{n} a_{i l} k_{l 1}^{+}, \\
k_{i 1}^{+}\left(t, d_{n} t / d_{1}\right)=0(i \neq n), \quad k_{i 1}^{+}(t, t)=a_{i 1} e^{a_{11} t} /\left(1-d_{i} / d_{1}\right)(i \neq 1),
\end{gathered}
$$

and from these equations we may deduce in the usual way

$$
\begin{aligned}
& k_{11}^{+}(t, t)=t e^{a_{11} t} \sum_{l=2}^{n} a_{1 l} a_{l 1} /\left(1-d_{l} / d_{1}\right), \\
& k_{n 1}^{+}\left(t, d_{n} t / d_{1}\right)=a_{n 1} e^{a_{n n} t} /\left(1-d_{n} / d_{1}\right) .
\end{aligned}
$$

For the $j$ th column of the kernel matrices we have from (3.9) for both $k^{+}$and $k^{-}$,

$$
\frac{\partial k_{i j}}{\partial t}+\frac{d_{i}}{d_{j}} \frac{\partial k_{i j}}{\partial s}=\sum_{l=1}^{n} a_{i l} k_{l j},
$$

while from (3.10) and (3.11) we may deduce

$$
\begin{gathered}
k_{i j}^{+}\left(t, d_{n} t / d_{j}\right)=0 \quad(i \neq n), \\
k_{i j}^{+}(t, t)-k_{i j}^{-}(t, t)=\frac{a_{i j} e^{a_{j j} t}}{\left(1-d_{i} / d_{j}\right)} \quad(i \neq j), \\
k_{i j}^{-}\left(t, d_{1} t / d_{j}\right)=0 \quad(i \neq 1),
\end{gathered}
$$

from which we can obtain

$$
\begin{gathered}
k_{n j}^{+}\left(t, d_{n} t / d_{j}\right)=-a_{n j} e^{a_{n n} t} /\left(1-d_{n} / d_{j}\right) \quad(j \neq n), \\
k_{j j}^{+}(t, t)-k_{j j}^{-}(t, t)=t e^{a_{j}, t} \sum_{\substack{l=1 \\
l \neq j}}^{n} \frac{a_{j l} a_{l i}}{\left(1-d_{l} / d_{j}\right)} \\
k_{1 j}^{-}\left(t, d_{1} t / d_{j}\right)=a_{1 j} e^{a_{j} t} /\left(1-d_{1} / d_{j}\right) \quad(j \neq 1) .
\end{gathered}
$$

For the last column of the kernel matrices, all elements of $k^{+}$are identically zero while the elements of $k^{-}$are nonzero only in the fan contained by $s=t$ and 
$s=d_{1} t / d_{n}$ and from (3.9), (3.10) and $(3.13)_{2}$ we have

$$
\begin{gathered}
\frac{\partial k_{i n}^{-}}{\partial t}+\frac{d_{i}}{d_{n}} \frac{\partial k_{i n}^{-}}{\partial s}=\sum_{l=1}^{n} a_{i l} k_{l n}^{-}, \\
k_{i n}^{-}\left(t, d_{1} t / d_{n}\right)=0(i \neq 1), \quad k_{i n}^{-}(t, t)=\frac{a_{i n} e^{a_{n n} t}}{\left(d_{i} / d_{n}-1\right)}(i \neq n),
\end{gathered}
$$

from which we may deduce

$$
\begin{gathered}
k_{1 n}^{-}\left(t, d_{1} t / d_{n}\right)=-\frac{a_{1 n} e^{a_{11} t}}{\left(d_{1} / d_{n}-1\right)} \\
k_{n n}^{-}(t, t)=t e^{a_{n n} t} \sum_{l=1}^{n-1} \frac{a_{n l} a_{l n}}{\left(d_{l} / d_{n}-1\right)} .
\end{gathered}
$$

Finally we observe that corresponding results along nonbounding characteristics can be obtained in terms of $\Delta_{i j} k_{\alpha \beta}^{ \pm}(t)$ defined by (3.15). For $\alpha \neq i$ we have immediately from (3.16)

$$
\Delta_{i j} k_{\alpha j}^{ \pm}(t)=0 \quad(i \neq j)
$$

while for $\alpha=i$ we have

$$
\Delta_{i j} k_{i j}^{ \pm}(t)=a_{i j} e^{a_{12} t} /\left(1-d_{i} / d_{\jmath}\right) \quad(i \neq j) .
$$

On the other hand if $i=j$ then we have

$$
\Delta_{\jmath \jmath} k_{j j}^{ \pm}(t)=t e^{a_{\jmath}, t} \sum_{\substack{l=1 \\ l \neq j}}^{n} \frac{a_{\jmath l} a_{l j}}{\left(1-d_{l} / d_{j}\right)}
$$

These results are derived as follows. From (3.9) and (3.15) we have

$$
\left(\frac{d}{d t}-a_{\imath i}\right) \Delta_{\imath j} k_{i j}^{ \pm}(t)=0 \quad(i \neq j),
$$

while if $i=j$ we obtain

$$
\left(\frac{d}{d t}-a_{\jmath j}\right) \Delta_{\jmath j} k_{j j}^{ \pm}(t)=e^{a_{j}, t} \sum_{\substack{l=1 \\ l \neq j}}^{n} \frac{a_{j l} a_{l j}}{\left(1-d_{l} / d_{j}\right)}
$$

and these differential equations can be solved for the discontinuities using the following argument to obtain a boundary condition at $t$ zero. For $i<j, d_{i}>d_{j}$ and as $t$ tends to zero, $k_{i j}^{-}$jumps from the value zero just above $s=d_{i} t / d_{j}$ to the value $a_{i j} /\left(d_{i} / d_{j}-1\right)$ just below this line (but above $s=t$ ) so that

$$
\lim _{t \rightarrow 0}\left[k_{i j}^{-}\left(t^{+}, d_{i} t / d_{j}\right)-k_{i j}^{-}\left(t^{-}, d_{i} t / d_{j}\right)\right]=a_{i j} /\left(1-d_{i} / d_{\jmath}\right)
$$


Similarly for $i>j, d_{i}<d_{j}$ and as $t$ tends to zero $k_{i j}^{+}$jumps from the value zero just below $s=d_{i} t / d_{j}$ to the value $a_{i j} /\left(1-d_{i} / d_{j}\right)$ just above this line (but below $s=t$ ) so that

$$
\lim _{t \rightarrow 0}\left[k_{i j}^{+}\left(t^{+}, d_{i} t / d_{j}\right)-k_{i j}^{+}\left(t^{-}, d_{i} t / d_{j}\right)\right]=a_{i j} /\left(1-d_{i} / d_{j}\right)
$$

and (6.9) evidently follows from (6.11), (6.13) and (6.14). Similarly

$$
\begin{gathered}
\lim _{t \rightarrow 0} \Delta_{j j} k_{j j}^{+}(t)=\lim _{t \rightarrow 0}\left[k_{j j}^{+}\left(t^{+}, t\right)-k_{j j}^{+}\left(t^{-}, t\right)\right]=\lim _{t \rightarrow 0} k_{j j}^{+}\left(t^{+}, t\right)=0 \\
\lim _{t \rightarrow 0} \Delta_{j j} k_{j j}^{-}(t)=\lim _{t \rightarrow 0}\left[k_{j j}^{-}\left(t^{+}, t\right)-k_{j j}^{-}\left(t^{-}, t\right)\right]=-\lim _{t \rightarrow 0} k_{j j}^{-}\left(t^{-}, t\right)=0
\end{gathered}
$$

which together with $(6.12)$ readily yields $(6.10)$.

\section{Conclusion}

For the fundamental problem of uncoupling systems of linear differential equations we have reviewed the situation relating to known uncoupling transformations for systems involving two dependent variables only. It appears that those systems for which uncoupling transformations have been derived are systems which can be transformed into a coupled system of the form (1.3) involving a single linear spatial operator $L$ only, for which the basic formal solution (1.4)-(1.7) is well established. Further for two dependent variables we have suggested two important coupled systems (2.19) and (2.20) for which formal solutions would have many applications. For more than two dependent variables, although the problem is unsolved, we have nevertheless presented in some detail the structure of solutions for the kernel matrices $k^{+}(t, s)$ and $k^{-}(t, s)$, and moreover we have utilised the governing system of partial differential equations to provide an elementary derivation of special values of the elements of these matrices along bounding characteristics. We have also indicated that further explicit representations of these kernel matrices hinges on inverting seemingly simple Laplace transforms of the form (5.23) for an arbitrary constant matrix $A$ and a diagonal matrix $D$.

\section{Acknowledgements}

The authors are grateful to both Noel Smyth and Bonnington Clarke for reading a preliminary version of this paper and making helpful comments. 


\section{References}

[1] E. C. Aifantis and J. M. Hill, "On the theory of diffusion in media with double diffusivity. Part I: Basic mathematical results", Quart. J. Mech. Appl. Math. 23 (1980) 1-21.

[2] J. M. Hill, "On the solution of reaction-diffusion equations", IMA J. Appl. Math. 27 (1981) 177-194.

[3] J. M. Hill and E. C. Aifantis, "On the theory of diffusion in media with double diffusivity. Part II: Boundary value problems", Quart. J. Mech. Appl. Math. 33 (1980) 23-41.

[4] A. I. Lee, "Mathematics of non-classical diffusion", Ph.D. Thesis, University of Wollongong, 1980.

[5] A. I. Lee and J. M. Hill, "A note on the solution of reaction-diffusion equations with convection", IMA J. Appl. Math. 29 (1982) 39-43.

[6] A. I. Lee and J. M. Hill, "On the general linear coupled system for diffusion in media with two diffusivities", J. Math. Anal. and Applics. 89 (1982) 530-557.

[7] A. McNabb, "An uncoupling procedure for a class of coupled linear partial differential equations", J. Austral. Math. Soc. B 26 (1985) 503-513.

[8] A. McNabb, "The kernel of operators uncoupling systems of linear partial differential equations", Applicable Analysis 23 (1986) 29-41.

[9] A. Pazy, Semigroups of linear operators and applications to partial differential equations (Springer-Verlag, New York, 1983). 